Check for updates

Cite this: RSC Adv., 2017, 7, 49498

\title{
Bone marrow mesenchymal stem cells inhibited bleomycin-induced lung fibrosis
}

\author{
Jiang Nan and Zhao Zhongyan (D) *
}

The present study was performed to evaluate the protective effect of bone marrow mesenchymal stem cells (BMSCS), TLR2-silencing BMSCS (BMSC ${ }^{\mathrm{TLR} 2-{ }^{-}}$), on bleomycin (BLM)-induced lung fibrosis and elucidate the critical role of TLR2 during the process. The BMSCs were isolated from adult male SD rats, and BMSC ${ }^{\text {TLR2-l- }}$ was constructed. Rats were intratracheally instilled with BLM. Then, $5 \times 10^{6}$ BMSCs and $5 \times 10^{6}$ BMSC $^{\text {TLR2-I- }}$ were injected into the tail vein 12 hours after the BLM challenge. All mice were sacrificed 21 days post BLM stimulation. As a result, treatment with BMSCs, but not with BMSC ${ }^{\mathrm{TLR} 2-1-}$, markedly ameliorated lung myeloperoxidase (MPO) activity, the wet-to-dry weight (W/D) ratio, and pulmonary histopathological alterations. Additionally, the protective effect of BMSCs might be attributed

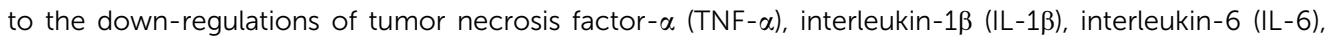
reactive oxygen species (ROS), and malondialdehyde (MDA) and the up-regulation of super-oxide dismutase (SOD). Furthermore, the administration of BMSC remarkably decreased the protein levels of MMP-3, MMP-9, TGF- $\beta, p-\operatorname{smad3}, p-I_{\kappa B}$, and $p-N F-\kappa B$, whereas the administration of BMSC ${ }^{\text {TLR2-l- }}$ exhibited no significant effect on the expressions of the abovementioned proteins. In conclusion, our results suggested that BMSCs exhibited a protective effect on BLM-induced pulmonary fibrosis, and TLR2 signaling might play an important role during this etiology.

Received 12th April 2017

Accepted 15th July 2017

DOI: 10.1039/c7ra03971a

rsc.li/rsc-advances basement membrane and matrix lipid peroxidation caused by oxidants. ${ }^{4}$

Mesenchymal stem cells were extracted from a variety of tissues and organs including cord blood, placenta, and bone marrow. During the past few decades, mesenchymal stem cell (MSC)-based cell therapy has been considered as an innovative treatment strategy. Bone marrow mesenchymal stem cells (BMSCs) are a population of multipotent stem cells that induce tissue regeneration. It was reported that BMSCs transplantation exhibited anti-fibrotic properties in lung injury., ${ }^{5,6}$ However, the underlying pathogenesis via which BMSCs treatment exerts a protective effect on BLM-induced pulmonary fibrosis is not fully understood. Therefore, the present study was conducted to further explore its potential mechanism in BLM-stimulated rats.

\section{Materials and methods}

\subsection{Reagents}

Bleomycin (BLM) was purchased from Sigma Chemical Co., (St. Louis, USA). Dexamethasone (Dex) was provided by Xiansheng Drug Store (Nanjing, China). The commercial kits for superoxide dismutase (SOD) and malondialdehyde (MDA) were supplied from Jiancheng Institute of Biotechnology (Nanjing, China). Tumor necrosis factor- $\alpha$ (TNF- $\alpha$ ), interleukin- 1 beta (IL$1 \beta$ ), and IL-6 enzyme-linked immunosorbent assay (ELISA) kits were obtained from BioLegend (San Diego, USA). All antibodies were produced by Cell Signaling Technology (Beverley, USA). 


\subsection{Animals}

SD rats (male, 180-220 $\mathrm{g}$ each), acquired from the Jiangning Qinglongshan Animal Cultivation Farm (Nanjing, China), were kept under standard laboratory conditions for 5 days before experiments. The animals were provided with water and standard chow ad libitum. All experimental protocols were conducted in accordance with animal handling following the dictates of the National Animal Welfare Law of China and in accordance with the guidelines of the National Institute of Nutrition, Hyderabad, India, and approved by the institutional ethical committee (IEC) of Jilin University.

\subsection{BMSCs isolation, culture, and characterization}

Adult male SD rats were used as BMSC donors. Under aseptic conditions, primary BMSCs were isolated from the femurs and tibias were removed. The central canal of the bone was washed with ice-cold PBS, and mononuclear cells were purified. Then, the samples were cultured at $37{ }^{\circ} \mathrm{C}$ under a humidified atmosphere with $5 \% \mathrm{CO}_{2}$ in complete DMEM/F-12 medium (Gibco) dissolved in $15 \%$ fetal bovine serum (Hyclone, South America), $100 \mathrm{IU} \mathrm{mL}^{-1}$ penicillin, and $100 \mathrm{IU} \mathrm{mL}^{-1}$ streptomycin (Amresco, USA). Non-adherent cells were removed via a medium change after 3 days. When colonies with a fibroblast-like appearance were observed, BMSCs were carried out at three-day intervals.

Surface marker expressions were detected by flow cytometry. In brief, the BMSCs $\left(1 \times 10^{6} / \mathrm{mL}\right)$ were trypsinized and fixed in $4 \%$ paraformaldehyde. CD34, CD44, and CD90 were taken as positive labeling for BMSCs characterization. ${ }^{7}$ An antibody against CD90 was obtained from Becton Dickinson (Mississauga, ON, Canada), and antibodies against CD11b, CD44, and CD45 were obtained from eBioscience (San Diego, CA, USA). All antibodies were conjugated with phycoerythrin.

To induce BMSCs to differentiate into adipocytes and osteocytes in vitro, specific differentiation media were applied at $37^{\circ} \mathrm{C}$ under a humidified atmosphere containing $5 \% \mathrm{CO}_{2}$. After three weeks, fat droplets and calcium were detected by staining with Oil Red $\mathrm{O}$ and Alizarin Red, respectively.

\subsection{Transient transfection}

Transient transfections were carried out using TLR2-shRNA plasmid (sc-108062, Santa Cruz, USA) according to the manufacturer's instructions. The BMSCs were obtained for analysis of TLR2 by Western blotting after a $48 \mathrm{~h}$ incubation.

\subsection{Experimental protocol for BLM-induced acute lung injury}

All rats were randomly divided into five groups: control group, BLM group, BLM + dexamethasone (Dex, $2 \mathrm{mg} \mathrm{kg}^{-1}$ ) group, $\mathrm{BLM}+\mathrm{BMSC}$, and BLM + $\mathrm{BMSC}^{\mathrm{TLR} 2-1-}$ group $(n=10)$. Rats were anesthetized by intraperitoneal injection of $3 \%$ chloral hydrate $\left(10 \mathrm{~mL} \mathrm{~kg}^{-1}\right)$ and then acutely cannulated for intratracheal instillation of BLM $\left(7.5 \mathrm{IU} \mathrm{kg}^{-1}\right.$ body weight in $0.25 \mathrm{~mL}$ sterile PBS). Animals in the control group were given PBS at the same volume. Then, $5 \times 10^{6} \mathrm{BMSCs}$ or $5 \times 10^{6} \mathrm{BMSC}^{\mathrm{TLR} 2-1-}$ were injected into the tail vein 12 hours after the BLM challenge. Simultaneously, the BLM + Dex $\left(2 \mathrm{mg} \mathrm{kg}^{-1}\right)$ group was treated with Dex $\left(2 \mathrm{mg} \mathrm{kg}^{-1}\right)$ dissolved in the medium. The control and BLM group were given an equal volume of culture medium. All mice were sacrificed 21 days post BLM stimulation. The blood was harvested from the abdominal aorta and centrifuged at $3000 \mathrm{~g}$ at $4{ }^{\circ} \mathrm{C}$ for $10 \mathrm{~min}$. Afterwards, the serum samples were maintained at $-80{ }^{\circ} \mathrm{C}$ for pending tests.

\section{$2.6 \mathrm{~W} / \mathrm{D}$ ratio assay}

Lung edema was determined by calculating the W/D ratio. Rats were sacrificed at 21 days after BLM exposure; subsequently, the right lungs were removed and wet weights were obtained. The lungs were then placed in an incubator at $60{ }^{\circ} \mathrm{C}$ for $48 \mathrm{~h}$ to remove all moisture, and the $\mathrm{W} / \mathrm{D}$ ratios were calculated.

\subsection{Polymorphonuclear (MPO) activity assay}

The pulmonary MPO activity was detected with assay kits according to manufacturer's instructions. Briefly, $100 \mathrm{mg}$ of lung tissues was homogenized and 5\% homogenate was incubated in a water bath for $15 \mathrm{~min}$ at $37^{\circ} \mathrm{C}$. Thereafter, the enzymatic activity was measured using a microplate spectrophotometer.

\subsection{Pulmonary histopathology}

The partial specimens of the lung tissues were fixed in normal $10 \%$ neutral-buffered formalin. After being dehydrated in graded alcohol, the sections were embedded in paraffin and subjected to microtome for haematoxylin and eosin (H\&E) staining. The pathological alteration was validated by two trained individuals who were blinded to the treatment under a light microscope.

\subsection{Determination of the antioxidant system}

The serum was obtained to assay the SOD and MDA levels. The operations were conducted according to the instructions on the commercial kits (Jiancheng Institute of Biotechnology, Nanjing, China).

\subsection{Cytokine measurement}

The levels of IL-6, IL- $1 \beta$, and TNF- $\alpha$ in serum were measured by ELISA kits according to the manufacturer's instructions. The optical densities (OD) were read at $450 \mathrm{~nm}$ using a microplate spectrophotometer.

\subsection{Detection of the intracellular ROS level in lung tissues}

Herein, $0.1 \mathrm{~g}$ lung tissues were homogenized in $1 \mathrm{~mL}$ normal saline. After centrifugation at $1500 \mathrm{~g}$ for $10 \mathrm{~min}$ at $4{ }^{\circ} \mathrm{C}$, the pellets were obtained and suspended. The intracellular redox state was determined by fluorescence probe 2,7-dichlorofluorescin diacetate kit (DCFH-DA, Sigma) according to the production instructions. The treated samples were washed with PBS three times and then incubated in $10 \mu \mathrm{M}$ DCFH-DA for $30 \mathrm{~min}$ at $37^{\circ} \mathrm{C}$ without light. The fluorescence intensity was proportional to the amount of ROS generated by the cells. The samples were analyzed by an enzyme-labeled instrument at an excitation wavelength of $488 \mathrm{~nm}$ and emission wavelength of $525 \mathrm{~nm}$. 


\subsection{Western blot}

Lung tissues were excised after sacrifice and stored at $-80{ }^{\circ} \mathrm{C}$ until homogenization. Generally, the samples were washed twice with PBS and extracted in a lysis buffer. Protein concentrations were detected by a BCA assay kit (Beyotime, Nanjing, China). Equal amounts of protein were separated by SDS-PAGE, and electrophoretically transferred onto polyvinylidene fluoride membranes (Millipore). After this, the membranes were blocked with $5 \%$ nonfat dry milk-TBST for $2 \mathrm{~h}$ and incubated with the primary antibodies overnight at $4{ }^{\circ} \mathrm{C}$. After washing, the blots were interacted with horseradish peroxidase (HRP)conjugated secondary antibodies for $2 \mathrm{~h}$ at room temperature.

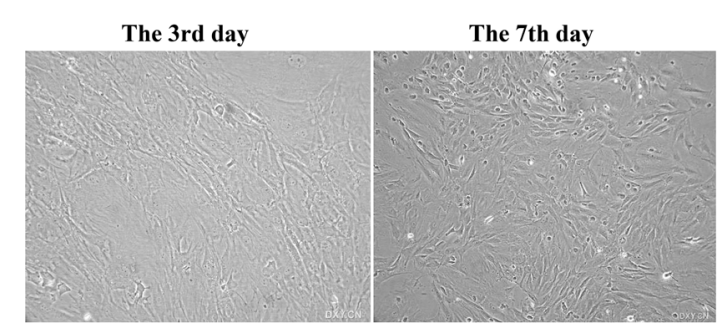

Fig. 1 The morphology of BMSC on the $3^{\text {rd }}$ and $7^{\text {th }}$ day after BMSC extraction.
Immunoreactivity was detected using an ECL plus Western blotting detection system (KeyGEN, Nanjing, China) and a gel imaging system (ChemiScope 2850, Shanghai, China).

\subsection{Statistical analysis}

The results are presented as mean \pm SD values. The differences between groups were analyzed by one-way ANOVA via a Tukey multiple comparison test. $P<0.05$ was considered as significant. All data calculations and analyses were conducted with the GraphPad Prism version 5.0.

\section{Results}

\subsection{Identification of isolated BMSCs in vitro}

In vitro, the isolated male BMSCs were adherent and displayed spindle-like shape during growth (Fig. 1). Similar to typical BMSCs, herein, the BMSCs were positive for mesenchymal lineage markers CD44, CD 34, and CD90 (Fig. 2), as observed via flow cytometric analysis. In addition, the BMSCs differentiated into adipogenic and osteogenic mesenchymal lineages after 21 days in differentiation media (Fig. 2C and D). These results demonstrated that the cells met the minimal criteria to be considered as BMSCs.
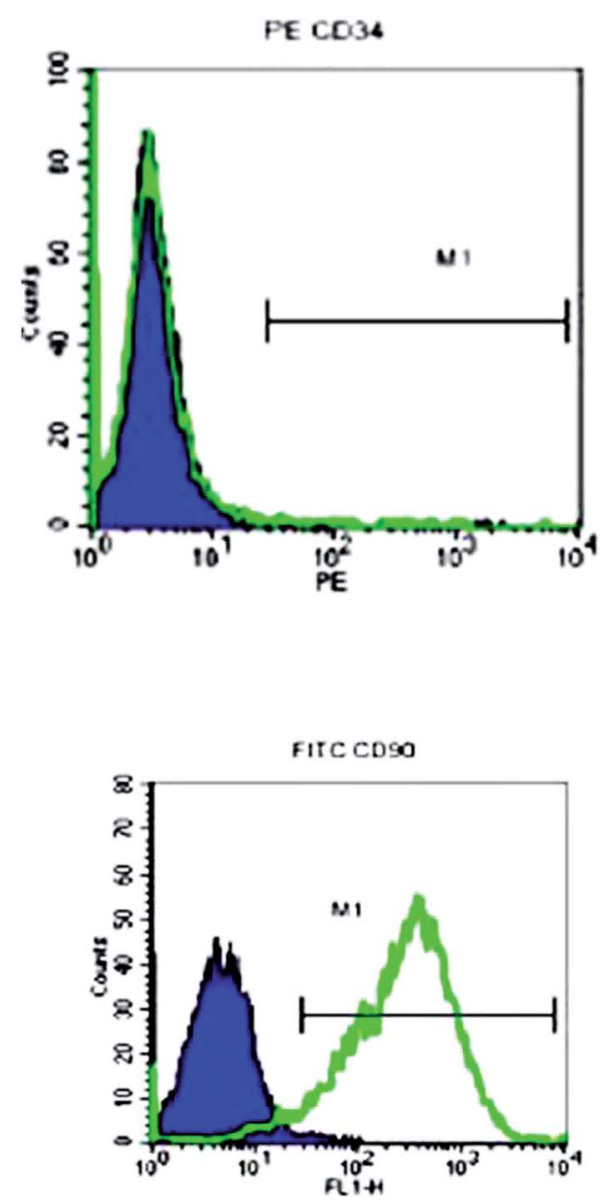

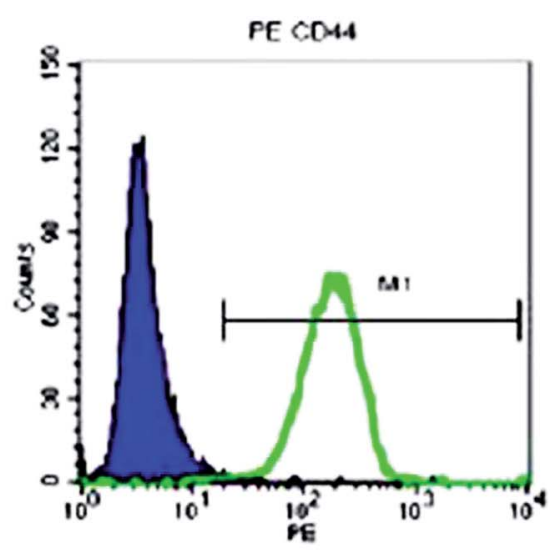

Fig. 2 Flow cytometry analysis of BMSC. 

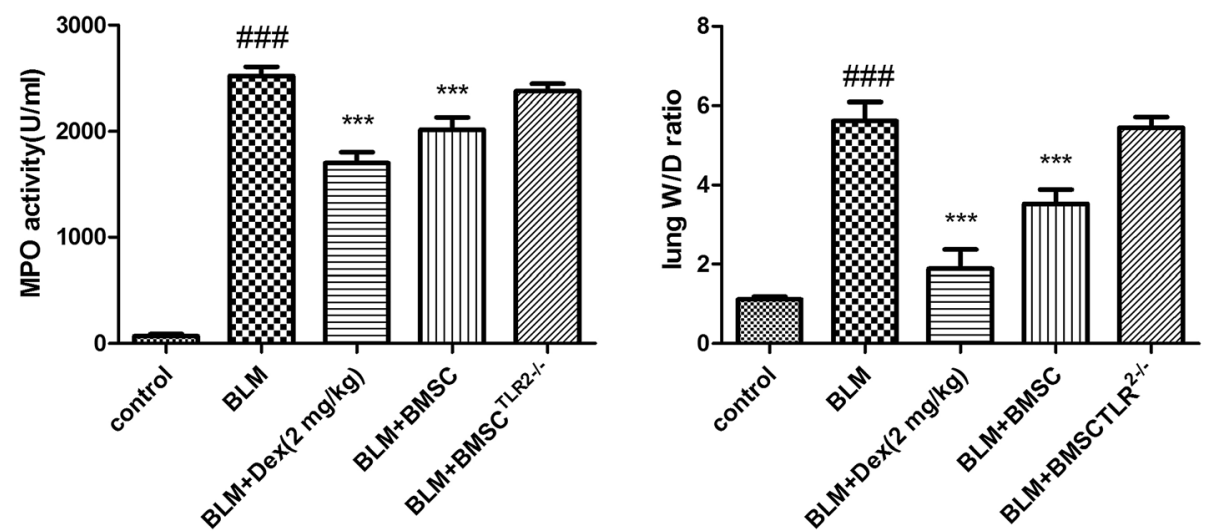

Fig. 3 The protective effect of BMSC and BMSC ${ }^{\text {TLR2-I- }}$ on MPO activity and W/D ratio in the lung tissues. BMSCS and BMSC ${ }^{\text {TLR2- } /-}$ were injected into the tail vein 12 hours after the BLM challenge. All mice were sacrificed 21 days after BLM stimulation. All values expressed as the mean \pm SD.

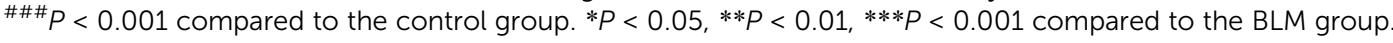

\subsection{Effects of BMSC and BMSC ${ }^{\mathrm{TLR} 2-/-}$ on lung MPO activity in BLM-induced rats}

MPO activity is a critical index for the accumulation of inflammatory cells in lungs. As shown in Fig. 3, it was proved that there was a remarkable increase of the MPO level in response to the BLM challenge. Both the BMSCs and dexamethanose reversed the abnormal alteration. However, the transfection of TLR2 seldom inhibited the MPO activity. These data indicated that BMSC was capable of inhibiting the MPO level in BLMinduced pulmonary fibrosis, but BMSC ${ }^{\mathrm{TLR} 2-/-}$ was not.

\subsection{Effects of BMSC and BMSC ${ }^{\mathrm{TLR} 2-/-}$ on lung $\mathrm{W} / \mathrm{D}$ ratio in BLM-induced rats}

The W/D (wet to dry) ratio was examined to show the content of edema in lung tissues. As illustrated in Fig. 2, the administration of BLM notably increased the lung W/D ratio. However, the treatment with BMSC or Dex markedly declined the magnitude of pulmonary edema, whereas the injection of $\mathrm{BMSC}^{\mathrm{TLR} 2-1-}$ seldom reduced the W/D ratio. The experimental data suggested that BMSC could affect the water content of lung tissues, whereas the silencing of TLR2 in BMSC blocked this beneficial activity.

\subsection{Effects of BMSC and BMSC ${ }^{\mathrm{TLR} 2-/-}$ on histopathological changes}

According to the results of HE staining, scarce pathological changes were observed in the control group, whereas the lung section in the BLM group showed a typical fibrotic appearance, infiltration of inflammatory cells, obvious cellular nodules, and collagen fibers. In contrast, the BMSC-treated group presented less inflammatory infiltration, thinner alveolar interstitial thickening, and smaller cellular nodules than the BLM group. Moreover, no obvious alteration was observed in the $\mathrm{BLM}^{\mathrm{TLR} 2-/-}$ group in lung specimens as compared to that in the BLM group. These results demonstrated that BMSC administration attenuated the histopathology changes in BLM-stimulated pulmonary fibrosis in rats (Fig. 4).

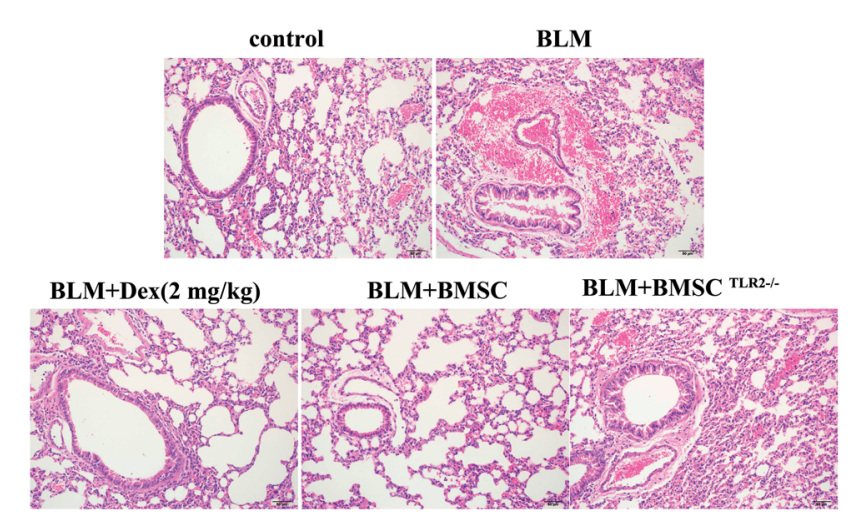

Fig. 4 The protective effect of BMSC and BMSC $\mathrm{TLR}^{\mathrm{TL}-/-}$ on the pathological condition in the lung tissues. BMSCs and BMSC ${ }^{\mathrm{TLR} 2-1-}$ were injected into the tail vein 12 hours after the BLM challenge. All mice were sacrificed 21 days after BLM stimulation. All values expressed as

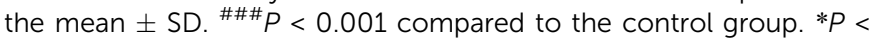
$0.05, * * P<0.01, * * * P<0.001$ compared to the BLM group.

\subsection{Effects of BMSC and BMSC ${ }^{\mathrm{TLR} 2-/-}$ on the levels of SOD} and MDA in serum of BLM-induced rats

The indicators including SOD and MDA were assessed to verify the anti-oxidative activity of BMSC and $\mathrm{BMSC}^{\mathrm{TLR} 2-/-}$. As shown in Fig. 5, it was confirmed that the rats exposed to BLM displayed a significant increase in the MDA concentration. However, treatments with BMSC and Dex $\left(2 \mathrm{mg} \mathrm{kg}^{-1}\right)$, but not with $\mathrm{BMSC}^{\mathrm{TLR} 2-/-}$, pronouncedly decreased the MDA content as compared to those in the BLM group.

In addition, BLM stimulation remarkably decreased the SOD activity. On the contrary, the treatment with BMSC and Dex (2 $\mathrm{mg} \mathrm{kg}{ }^{-1}$ ) evidently restored the SOD activity, whereas BMSC $^{\text {TLR2-1- }}$ administration did not influence the SOD level as compared to those in the BLM group.

\subsection{Effects of BMSC and BMSC ${ }^{\mathrm{TLR} 2-/-}$ on intracellular ROS production in lung tissues of BLM-induced rats}

The content of ROS in response to BLM was measured using a DCFH-DA probe. As shown in Fig. 4, the BLM group exhibited 

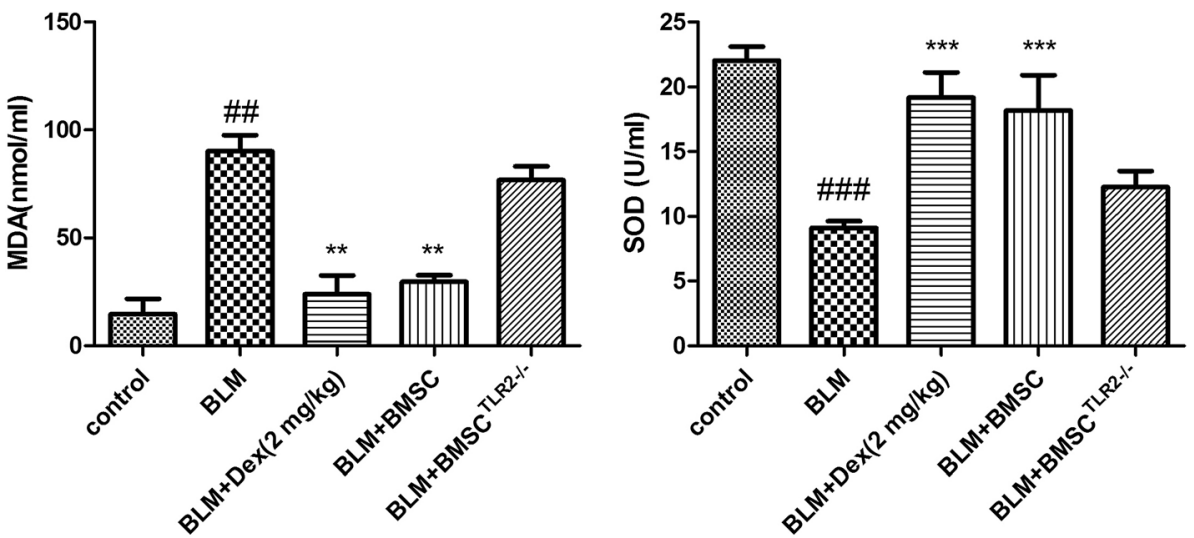

Fig. 5 The protective effect of BMSC and BMSC ${ }^{\text {TLR2-I- }}$ on the levels of SOD and MDA in serum. BMSCs and BMSC ${ }^{\text {TLR2-I- }}$ were injected into the tail vein 12 hours after the BLM challenge. All mice were sacrificed 21 days after BLM stimulation. All values expressed as the mean \pm SD. ${ }^{\# \# \# ~} P<$ 0.001 compared to the control group. ${ }^{*} P<0.05, * * P<0.01,{ }^{* * *} P<0.001$ compared to the BLM group.

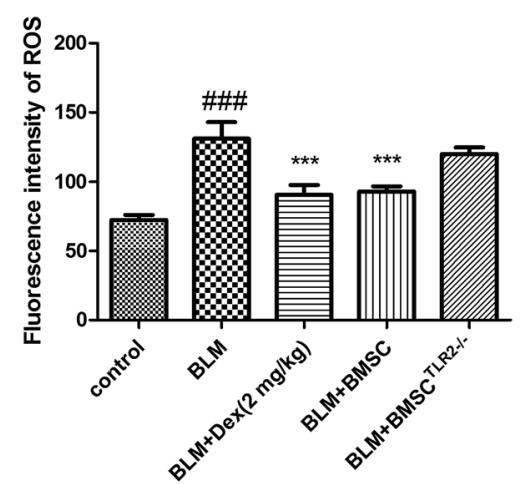

Fig. 6 Effects of BMSC and BMSC ${ }^{\text {TLR2-1- }}$ on intracellular ROS production in lung tissues of BLM-induced rats. All mice were sacrificed 21 days after BLM stimulation. All values expressed as the mean \pm

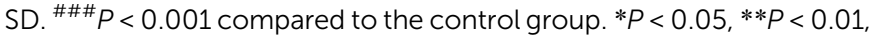
$* * * P<0.001$ compared to the BLM group.

significantly elevated ROS level as compared to the control group; the administration of BMSC and Dex notably decreased the ROS production, whereas the $\mathrm{BMSC}^{\mathrm{TLR} 2-/-}$ scarcely reduced the ROS level. Based on these results, it was proven that BMSC could inhibit the generation of ROS, and TLR2 silencing downregulated this effect.

\subsection{Effects of BMSC and BMSC ${ }^{\text {TLR2-l- }}$ on inflammatory cytokines in serum of BLM-induced rats}

Inflammatory cytokines are a major factor accounting for the intervention of BLM-induced pulmonary fibrosis model. According to the results, the contents of IL-1 $\beta$, IL- 6 , and TNF$\alpha$ were elevated after BLM exposure (Fig. 6). Treatment with BMSC and Dex $\left(2 \mathrm{mg} \mathrm{kg}^{-1}\right)$ reduced the production of inflammatory cytokines as compared to the case of BLM group. However, little therapeutic efficacy of $\mathrm{BMSC}^{\mathrm{TLR} 2-1-}$ on TNF- $\alpha$, IL-6, and IL-1 $\beta$ was observed. The analytical results demonstrated that BMSC, but not BMSC ${ }^{\text {TLR2-I- }}$ treatment, suppressed inflammatory mediators in BLM-stimulated rats.

\subsection{Effects of BMSC and BMSC ${ }^{\text {TLR2 }-/-}$ on the protein} expressions of MMP-3, MMP-9, TGF- $\beta$, smad3, and NF- $\mathrm{\kappa B}$ pathway

The protein expressions of MMP-3, MMP-9, TGF- $\beta, p-\operatorname{smad} 3$,

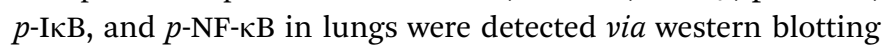
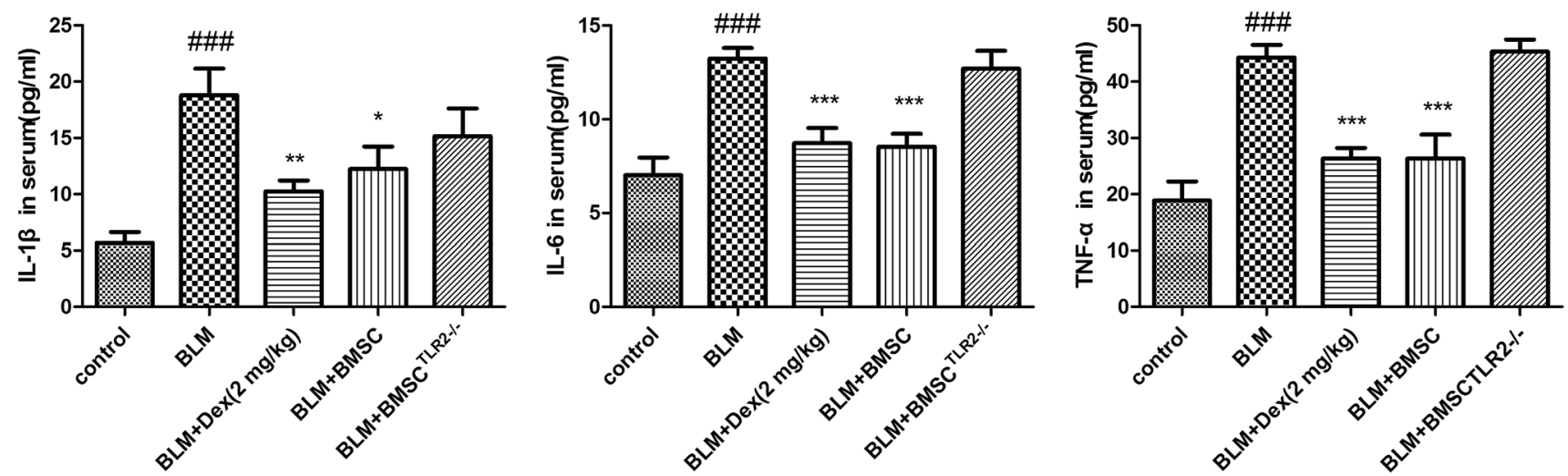

Fig. 7 The protective effect of BMSC and BMSC ${ }^{\text {TLR2-I- }}$ on the contents of IL-1 $\beta, I L-6$ and TNF- $\alpha$ in serum. BMSCS and BMSC ${ }^{\text {TLR2-I- }}$ were injected into the tail vein 12 hours after the BLM challenge. All mice were sacrificed 21 days after BLM stimulation. All values expressed as the

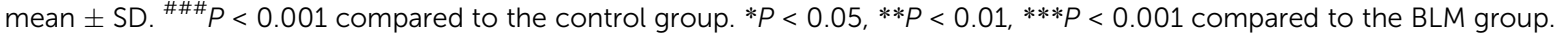




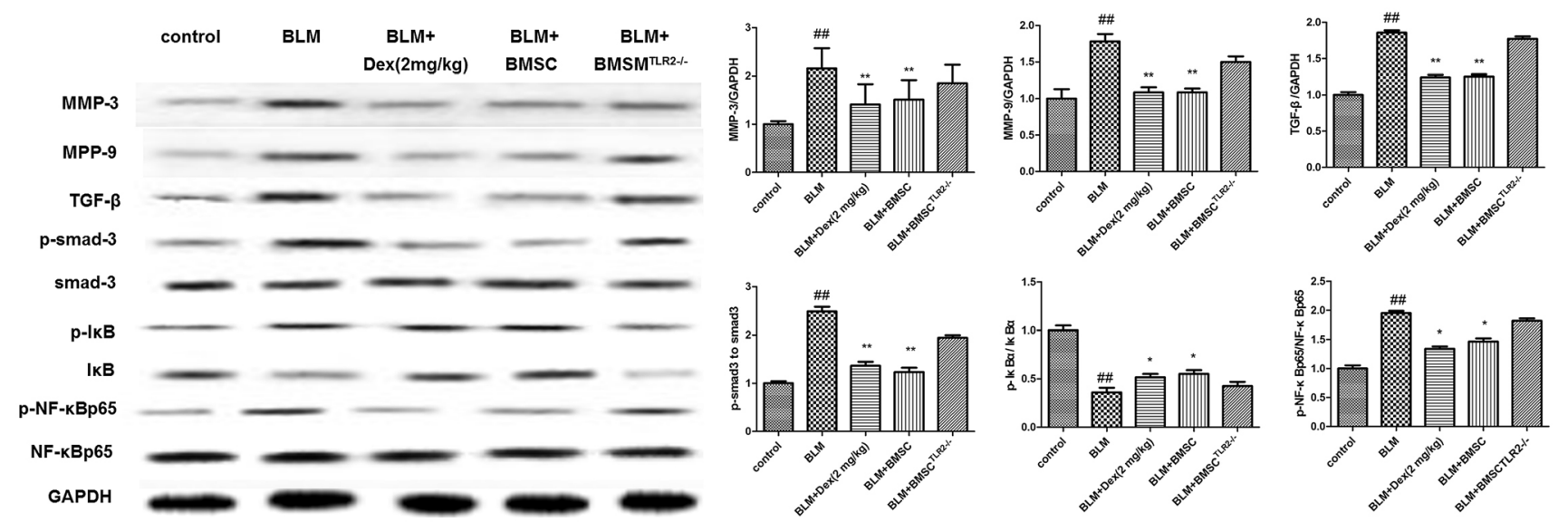

Fig. 8 The protective effect of BMSC and BMSC ${ }^{T L R 2-/-}$ on the expressions of MMP-3, MMP-9, TGF- $\beta, p-s m a d 3, p-I \kappa B$ and $p-N F-\kappa B$ in lung tissues. BMSCs and BMSC ${ }^{\mathrm{TLR} 2-1-}$ were injected into the tail vein 12 hours after the BLM challenge. All mice were sacrificed 21 days after BLM stimulation. All values expressed as the mean \pm SD. ${ }^{\# \# \# ~} P<0.001$ compared to the control group. $* P<0.05, * * P<0.01, * * * P<0.001$ compared to the BLM group.

assay. As revealed in Fig. 7, exposure to BLM obviously increased the protein expressions of MMP-3, MMP-9, TGF- $\beta$, $p$-smad3, $p$-I $\kappa \mathrm{B}$, and $p$-NF- $\kappa \mathrm{B}$. After treatment with BMSC and Dex $\left(2 \mathrm{mg} \mathrm{kg}^{-1}\right)$, these protein expressions were efficiently inhibited as compared to those in the BLM group. On the contrary, the administration of $\mathrm{BMSC}^{\mathrm{TLR} 2-1-}$ presented no significant effect on the expressions of MMP-3, MMP-9, and TGF- $\beta$ and the phosphorylations of $\operatorname{smad} 3, \mathrm{I} \kappa \mathrm{B}$, and NF- $\kappa \mathrm{B}$ as compared to the case of the BLM groups (Fig. 8).

\section{Discussion}

Numerous evidence suggests that BMSCs protect lungs against lung fibrotic lesions by preventing the transformation of extracellular matrix and fibroblasts. ${ }^{8}$ Our study also confirmed that treatment with BMSCs attenuated the pathological changes, lung MPO activity, and W/D ratio in BLM-stimulated lung injury.

Bleomycin is an antitumor drug with cytotoxicity accompanied by free radicals, which results in DNA damage leading to cell death. The stimulation of bleomycin is characterized by the development of pulmonary fibrosis, which limits its clinical use. Intratracheal administration of bleomycin (BLM) has been widely applied for studying the pathogenesis and intervention of pulmonary fibrosis in animals. ${ }^{9}$ It is noteworthy that its major toxicity target organ is lung since this tissue selectively lacks an enzyme that hydrolyzes bleomycin and prevents the metabolic products from binding to DNA or metal ions. ${ }^{10}$ We observed significant pulmonary injuries and dysfunction after a BLM challenge, evidenced by deterioration of histopathology, and increased MPO activity and W/D ratio. Edema is a pivotal symptom of partial and systemic inflammation. MPO produces superabundant oxidants conducive to pulmonary damage under inflammatory circumstances. Our results confirmed that the BMSC administration could attenuate the dysfunctions in lung tissues, but BMSC ${ }^{\text {TLR2-/- }}$ could not.

MDA, the reliable index of oxidative stress, is the end product of polyunsaturated fatty acid and reflects cell damage attributed to reactive oxygen metabolites in pulmonary dysfunction. ${ }^{11}$ The endogenous anti-oxidative enzyme SOD plays a critical role in protecting lung tissues against ROS activation by oxidative stress. $^{12}$ Our results suggested that the BMSC therapeutic method relieved lipid oxidation, but the $\mathrm{BMSC}^{\mathrm{TLR} 2-/-}$ did not affect the oxidative stress caused by BLM exposure.

TNF- $\alpha$ participates in the induction of inflammation, upregulation of TGF- $\beta$, and the proliferation of fibroblasts ${ }^{\mathbf{1 3 , 1 4}}$ IL-1 $\beta$ is responsible for increasing collagen synthesis induced chronic peritoneal fibrosis in patients. ${ }^{\mathbf{1 5}}$ The blockade of the IL-6 signaling proves to be a beneficial effect on lung fibrosis. ${ }^{16}$ To evaluate the anti-inflammatory effects of BMSC and $\mathrm{BMSC}^{\mathrm{TLR} 2-1-}$ treatment on inflammation of pulmonary fibrosis induced by BLM, we measured the serum contents of the key inflammatory cytokines including IL-1 $\beta$, IL-6, and TNF- $\alpha$. These cytokines were significantly reduced in the BMSC-treated animals, whereas they were not very significant in the $\mathrm{BMSC}^{\mathrm{TLR} 2-/-}$ group.

The fibroblast differentiation, which was mediated by the transforming growth factor- $\beta$ (TGF- $\beta$ )/Smad, is associated with the development of lung fibrosis. TGF- $\beta$ serves as a profibrotic cytokine inducing the structural alteration of airway remodeling. TGF- $\beta$ is secreted by epithelial cells and fibroblasts in airway tissue and implicated with subepithelial fibrosis. ${ }^{17}$ The TGF- $\beta$ signaling is transduced by the phosphorylation intracellular effectors Smad3. ${ }^{18}$ The bone regeneration and repair involve the secretion of matrix metalloproteinases (MMPs) by BMSCs to degrade the surrounding extracellular matrix (ECM). Evidence has emerged indicating that MMP-3 has a wide range of actions affecting fibrinolysis and angiogenesis. ${ }^{19} \mathrm{MMP}-9$ is proposed to be related to the neutrophils inflammatory reactions of lung tissues. ${ }^{20} \mathrm{NF}-\kappa \mathrm{B}$, activated by the phosphorylation and degradation of $\mathrm{I} \kappa \mathrm{B}$, is an essential transcription factor involved in immune and inflammatory responses. ${ }^{21} \mathrm{NF}-\kappa \mathrm{B}$ controls the expression of various mediators including TNF- $\alpha$, IL-1 $\beta$, IL-6, and the MMP family. ${ }^{22}$ Our results showed that treatment with BMSC exhibited an 
inhibitory effect on MMP-3, MMP-9, TGF- $\beta, p$-smad3, $p$-I $\kappa$ B, and $p$-NF- $\kappa \mathrm{B}$ in lung tissues, whereas the treatment with BMSC $^{\text {TLR2-1- }}$ showed no significant effect on these protein levels.

In conclusion, the present study demonstrated that treatment with BMSC exhibited a protective effect on BLM-induced pulmonary fibrosis via anti-fibrotic, anti-oxidative, and antiinflammatory activities possibly through the TGF- $\beta / \mathrm{Smad} / \mathrm{NF}$ $\kappa \mathrm{B}$ pathway. Additionally, TLR2 might play a pivotal role in this progression. Further research is warranted before clinical application.

\section{Acknowledgements}

The Bethune special funds of science and technology development plans of Jilin province.

\section{References}

1 Y. Shi, Q. Chen, H. Yan and W. Gu, Int. Immunopharmacol., 2016, 41, 116-121.

2 B. B. Moore and C. M. Hogaboam, Am. J. Physiol.: Lung Cell. Mol. Physiol., 2008, 294, L152-L160.

3 Q. Wang, R. Wen, Q. Lin, N. Wang, P. Lu and X. Zhu, Dig. Dis. Sci., 2015, 60, 3329-3339.

4 C. Yuan, C. Qian, L. Jing, F. H. Li, Y. Y. Tao and C. H. Liu, Chin. J. Integr. Med., 2009, 15, 435.

5 J. Xu, L. Li, J. Xiong, Y. Zheng, Q. Ye and Y. Li, Ann. Clin. Lab. Sci., 2015, 45, 292-300.

6 F. F. Cruz and P. R. M. Rocco, J. Stem Cell Res. Ther., 2015, 6, 130.

7 M. Dominici, K. Le Blanc, I. Mueller, I. Slaper-Cortenbach, F. Marini, D. Krause, R. Deans, A. Keating, D. Prockop and E. Horwitz, Cytotherapy, 2006, 8, 315-317.

8 L. H. Dong, Y. Y. Jiang, Y. J. Liu, S. Cui, C. C. Xia, C. Qu, X. Jiang, Y. Q. Qu, P. Y. Chang and F. Liu, Sci. Rep., 2015, 5, 8713 .
9 J. Nikbakht, A. A. Hemmati, A. Arzi, M. T. Mansouri, A. Rezaie and M. Ghafourian, Pharmacol. Rep., 2015, 67, 1061-1067.

10 A. E. Filderman, L. A. Genovese and J. S. Lazo, Biochem. Pharmacol., 1988, 37, 1111-1116.

11 T. Chen, R. Wang, W. Jiang, H. Wang, A. Xu, G. Lu, Y. Ren, Y. Xu, Y. Song, S. Yong, H. Ji and Z. Ma, Inflammation, 2016, 39, 483-492.

12 R. Zhou, F. Luo, H. Lei, K. Zhang, J. Liu, H. He, J. Gao, X. Chang, L. He, H. Ji, T. Yan and T. Chen, J. Ethnopharmacol., 2016, 193, 643-651.

13 G. S. Warshamana, M. Corti and A. R. Brody, Exp. Mol. Pathol., 2001, 71, 13-33.

14 T. A. Wynn, J. Exp. Med., 2011, 208, 1339.

15 M. S. Wilson, S. K. Madala, T. R. Ramalingam, B. R. Gochuico, I. O. Rosas, A. W. Cheever and T. A. Wynn, J. Exp. Med., 2010, 207, 535-552.

16 T. T. T. Le, H. Karmoutyquintana, E. Melicoff, T. T. T. Le, T. Weng, N. Y. Chen, M. Pedroza, Y. Zhou, J. Davies and K. Philip, J. Immunol., 2014, 193, 3755.

17 A. Leask and D. J. Abraham, FASEB J., 2004, 18, 816.

18 K. Shi, J. Jiang, T. Ma, J. Xie, L. Duan, R. Chen, P. Song, Z. Yu,

C. Liu and Q. Zhu, Int. J. Clin. Exp. Med., 2014, 7, 2645-2650.

19 A. G. Richter, S. Mckeown, S. Rathinam, L. Harper, P. Rajesh,

D. F. Mcauley, R. Heljasvaara and D. R. Thickett, Thorax, 2009, 64, 156-161.

20 H. Lemjabbar, P. Gosset, E. Lechaptzalcman, M. L. Francomontoya, B. Wallaert, A. Harf and C. Lafuma, Am. J. Respir. Cell Mol. Biol., 2012, 20, 903-913.

21 Q. Jiang, M. Yi, Q. Guo, C. Wang, H. Wang, S. Meng, C. Liu, Y. Fu, H. Ji and T. Chen, Int. Immunopharmacol., 2015, 29, 370-376.

22 T. Chen, Y. Mou, J. Tan, L. Wei, Y. Qiao, T. Wei, P. Xiang, S. Peng, Y. Zhang, Z. Huang and $\mathrm{H}$. Ji, Int. Immunopharmacol., 2015, 25, 55-64. 